\title{
ARTICLE
}

\section{Development of real-time Bayesian data assimilation system for off-site consequence assessment}

\author{
Radek Hofman*, Václav Šmídl and Petr Pecha \\ Institute of Information Theory and Automation, Academy of Sciences of the Czech Republic \\ Pod Vodárenskou věži 4, 18208 Praha 8, Czech Republic
}

\begin{abstract}
The task of decision support in the case of a radiation accident is to provide up-to-date information on the radiation situation, prognosis of its future evolution and possible consequences. The reliability of predictions can be significantly improved using data assimilation, which refers to a group of mathematical methods for an efficient combination of observed data with a numerical model. This paper concerns application of advanced data assimilation methods in the off-site consequence assessment when radionuclides are released into the atmosphere. Our goal is to develop a decision support system with automated real-time assessment of radiation situation that does not underestimate its uncertainty. To achieve this goal we employ particle filtering - a Bayesian approach suitable for sequential processing of a stream of measurements incoming from a monitoring network. The output of this assimilation procedure is the whole posterior probability density of a quantity of interest. This allows for reasoning in a probabilistic manner. The system is equipped with tools for this type of assessment and the focus is paid to ease of use during its development. Performance of the system is demonstrated on a simulation scenario.
\end{abstract}

Keywords: data assimilation; atmospheric dispersion modeling; decision support system; sequential Monte Carlo; probabilistic assessment

\section{Introduction}

Release of radioactive material into the atmosphere is the worst case scenario of any accident in a nuclear power plant. It is an extremely rare event, however with severe consequences for potentially many people living in proximity of the power plant. In the case of such a release the risk evaluation based on modeling of spatial and temporal distribution of radionuclides is essential for countermeasures planning. These predictions can be significantly improved using data assimilation. Also known as statistical inference, data assimilation refers to a group of mathematical methods for estimation of state of a dynamic system by the means of combining multiple information sources. Typically, numerical model of the system under investigation is corrected with observational data.

One of the fundamental tasks in the case of a radiation accident is the prediction of radiation situation during the early phase when the radionuclides released into the atmosphere are dispersed by the wind and turbulence. Recent advances in the field of dispersion modeling and statistical inference have shown great promise in reduction of uncertainty in the source term and meteorological inputs. This is achieved by

*Corresponding author. Email: hofman@utia.cas.cz systematic comparison of modeled radiological quantities and those observed via stationary "Radiation Monitoring Network (RMN)" and mobile groups [1]. Improved estimates may in turn be used as inputs to long- and short-range atmospheric dispersion models, with a view to increasing the reliability of decision-making resulting in higher efficiency of countermeasures.

This problem has been already addressed using different methods. The most notable are spatial interpolation [2], variational methods [3], Kalman filtering [4] and Bayesian estimation [5, 6]. Bayesian estimation, contrary to previously mentioned methods, provides not only point estimates but full probability distributions of unknown quantities conditioned on available data (posterior distributions). Using these distributions, decision makers' questions can be formulated in a probabilistic manner, e.g.: "What is the probability of exceeding a given dose limit at a particular location?"

We aim to design a Bayesian data assimilation system for autonomous assessment of radiation situation during the early phase of an accident. Since the very beginning of the release, RMN provides gamma dose rate measurements at regular intervals. Our goal is to process this stream of measurements from RMN and analyze radiation situation in real time. 
In this paper we briefly present the system, chosen data assimilation methodology, target data assimilation scenarios and the prospect of future development.

\section{Developed decision support system}

\subsection{Atmospheric dispersion modeling}

Atmospheric dispersion models represent the core of the system. Currently, "Segmented Gaussian Plume Model (SGPM)" [7] and a Lagrangian puff model are integrated. While the puff model is still under development, SGPM has been already certified for emergency management use in the Czech Republic. It has been developed for more than 10 years and successfully validated against COSYMA and MACCS codes. Besides activity concentration fields of more than 120 radionuclides the model also evaluates consequent radiological quantities: doses from cloudshine (with respect to geometry of the plume), groundshine and inhalation (even from resuspension). What is more, these quantities are evaluated for 6 age groups and different body organs. Subsequent food-chain model simulates propagation of radionuclides through the environment towards the human body.

Both models can run independently on the data assimilation part of the system. Spatially homogeneous meteorological data can be entered by users or spatially variable numerical weather forecasts can be fetched using a meteorological preprocessor connected to a database of meteorological forecast data (ALADIN and WRF models). Example of SGPM output visualized as an overlay on Google Maps is in Figure 1.

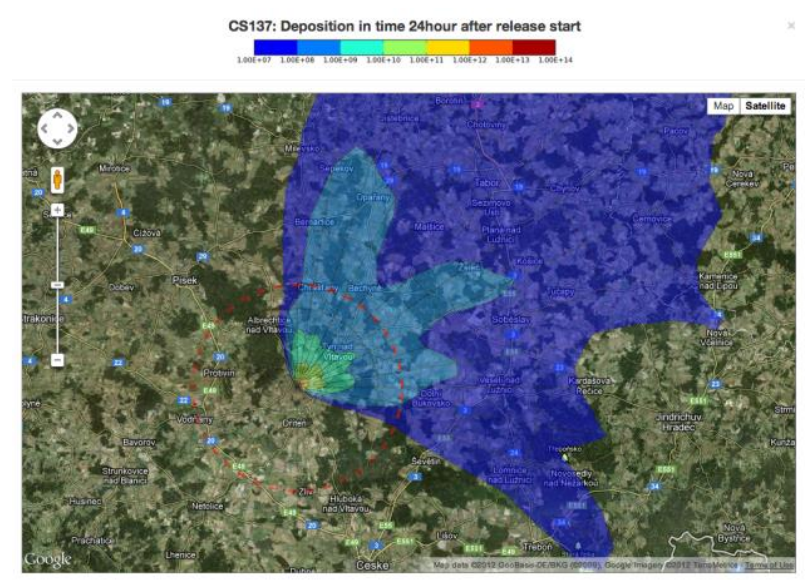

Figure 1. Illustration of SGPM output.

\subsection{Data assimilation methodology}

We propose a data assimilation algorithm based on particle filtering, which results from sequential Monte Carlo. Particle filter approximates the posterior distribution of estimated variables using weighted samples from so called proposal function representing "the first guess" of the posterior [8]. The weights are sequentially evaluated on basis of correspondence of samples with incoming measurements. In our data assimilation procedure the samples are outputs from dispersion models with different initial settings.

Basic principle of the algorithm is illustrated in Figure 2. Here, the "real" reference release is represented with color isopleths. Radiation monitoring network providing gamma dose rate measurements is denoted with red triangles. In the real assimilation scenarios we simultaneously propagate hundreds or thousands of dispersion models. Here, for sake of simplicity, the approach is illustrated on four instantaneous puffs $P 1-P 4$. We track them for the first three time steps. Puffs vary in the source term and are propagated using different wind speed and direction. In the first time step, puffs $P 2$ and $P 3$ cover receptors (monitoring sites) influenced with the release and obtain high weights. During re-sampling step, puffs with high weights have a high probability to be copied and propagated in next time step (puffs $P 2$ and $P 3$ ), while the others are discarded and replaced with the new ones similar to those re-sampled. In the second time step, three puffs obtain high weights and are re-sampled: $P 2.2$, $P 2.3$ and P3.1. In the third time step, puffs $P 2.2 .1$ and $P 3.1 .1$ are advected to areas unaffected with the release, obtain small weights and are discarded. Surviving puffs $P 2.3 .1$ and P2.3.2 best fitting available gamma dose rate measurements are propagated further and so on [9].

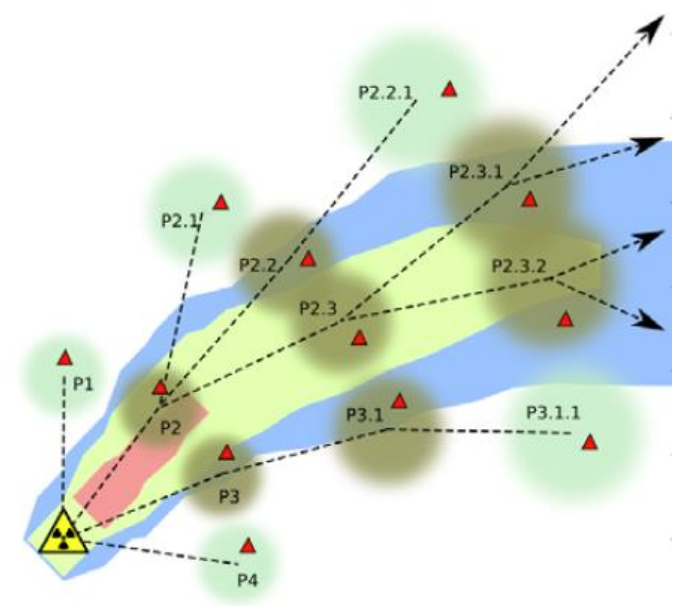

Figure 2. Illustration of the basic principle of the data assimilation method based on particle filtering.

The algorithm is applicable to all parameterized dispersion models. Proposal function was tailored in order to maximize efficiency of the algorithm in such a real-time application [1]. Besides the source term and meteorological conditions, the set of estimated variables can also parameterize other important processes and quantities influencing propagation of radionuclides, like rates of dry and wet deposition, dispersion coefficients.

\subsection{Modes of operation}

The system is intended to operate in two regimes: (i) autonomous on-line regime; and (ii) supervised off-line regime.

In the on-line regime, measured radiological data and 
meteorological data are gathered on-line. Since this regime is fully autonomous, incoming measurements are continuously evaluated. Data assimilation procedure is automatically triggered if abnormal values are detected and prediction of spatial-temporal distribution of radionuclides is successively corrected real-time using incoming measurements.

In the off-line regime, radiological measurements are entered by user. This regime can be used for retrospective assessment of the release, performing what-if analyses and for educational and training purposes. It can be also applied to testing of different RMN configurations and assessing its capability to detect releases under various circumstances.

\section{Demonstration of the system}

The system is implemented using client-server architecture. Users can connect to it using web and desktop clients. The focus is paid to ease of use and illustrative visualization of data assimilation results.

To demonstrate the system we present an assimilation scenario with a simulated release of radionuclide Ar-41 with unknown magnitude, start and duration. This scenario demonstrates the performance of the system during on-line continuous monitoring. The release is assumed to occur from the Czech NPP Temelín. RMN in this experiment has the same topology as the real RMN currently present on site. It is comprised of two rings of receptors. The goal is to estimate the magnitude of release and biases of forecasted wind speed and wind direction using gamma dose rate measurements. In this scenario, continuous release is modeled as a sequence of instantaneous puffs released every 10 minutes. All estimated quantities are time variant with step size 10-minute-puff.

In Figure 3-top we see the cloudshine dose estimate based on biased meteorological data and a first guess of release magnitude. Reference release used for simulation of perturbed measurements is in Figure 3-midddle. Expected value of cloudshine dose estimated using data assimilation of measurements up to time 4 hours after the release start is in Figure 3-bottom. We observe that the biases of meteorological data were successfully estimated and accounted for.

Figure 4 shows estimates of released activity in time. Black dashed line represents the "true" magnitude of release - two consecutive releases of duration 1 and 2 hours, respectively, separated by 1 hour long period when no release occurs. Blue boxplots represent samples from posterior distribution at each time step. We observe that the system successfully identified the duration of the release (time 0 denotes start of the first release) and released activities. The "true" values fall within the interval of minimum and maximum estimates denoted by the gray band. When no release occurs, the estimated magnitudes of release are equivalent to the threshold of detectability of the receptors given by the natural background radiation.
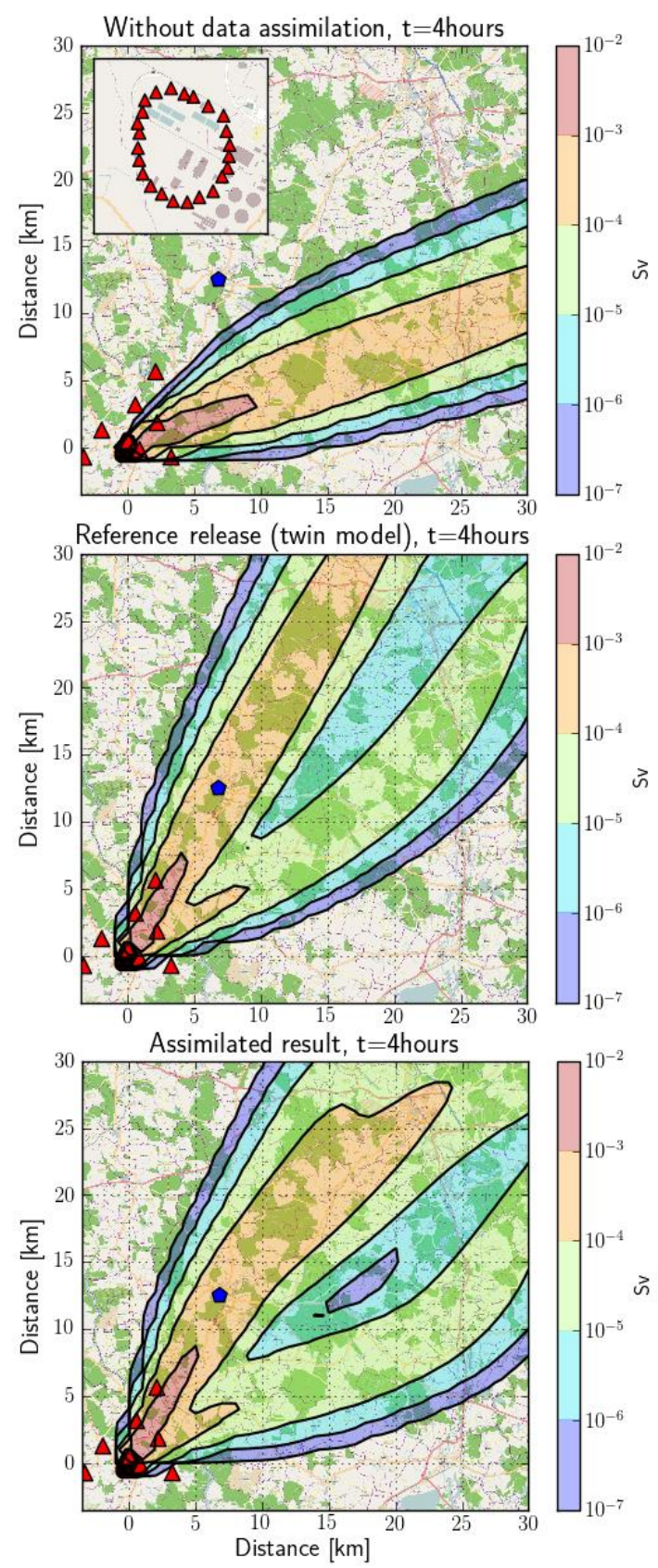

Figure 3. Top: Simulation of cloudshine dose based on nominal data without data assimilation. Middle: Twin model representing the true release. Bottom: Expected value of dose after data assimilation procedure. Blue pentagon is a point of interest, an inhabited place, where want to estimate the dose distribution and use it for countermeasures planning. Radiation monitoring network is denoted by red triangles, detail of the first ring of receptors is in left top corner of the top figure.

Using the dispersion model and distributions of its variables, probabilistic approach enables to estimate the distribution of different radiological quantities like 3-D activity concentration fields and consequently doses from the cloud, deposition and inhalation (using ground-level activity concentrations) in an arbitrary set of points of interest (e.g. inhabited places). Figure 5 shows posterior density (green histogram) of time 
integrated cloudshine dose of up to 4 hours since the release start which is in a good agreement with the "true" value of the reference release (vertical blue line).

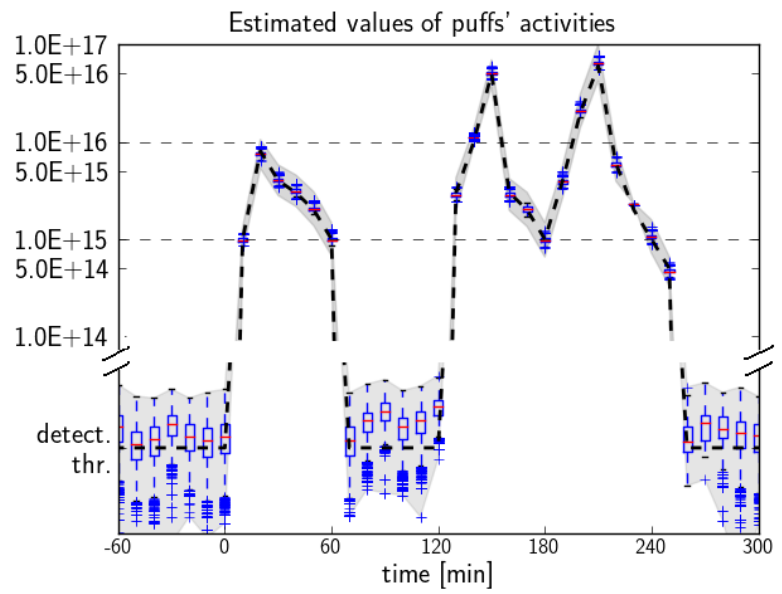

Figure 4. Estimates of released activity during continuous monitoring in on-line regime. The estimates (blue boxplots) are in good agreement with the true values (black dashed line).

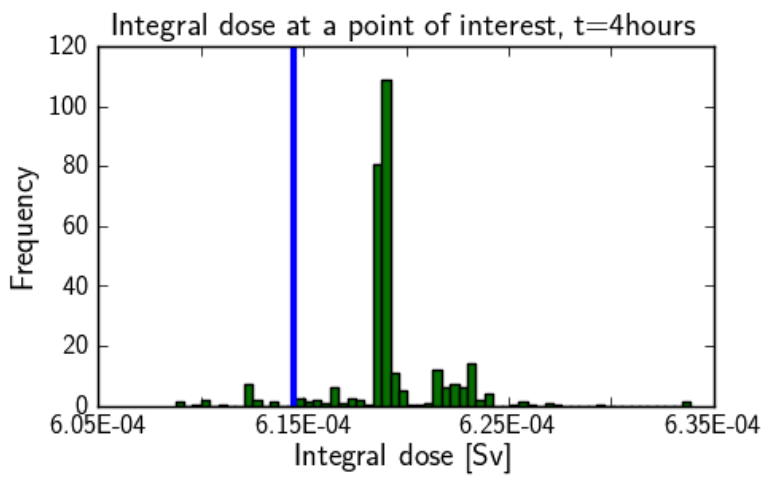

Figure 5. Histogram of total committed dose at a point of interest - its location is denoted in Figure 3 as a blue pentagon.

\section{Conclusion}

The research has demonstrated that the probabilistic approach provides useful insights into the problem of radiation situation assessment in the case of an accident with off-site consequences. Data assimilation system based on particle filtering has a potential to be used for real world emergency response in the near future. Its development (in cooperation with the Czech National Radiation Protection Institute) pushes real-time data assimilation in the Czech Republic towards operational application.

The system was demonstrated on an artificial scenario with release of a single pollutant. This would be a realistic assumption in the case of a non-radioactive accident, e.g. spreading of a toxic agent in urban areas. For successful application in radiation protection it must be extended for source terms with more nuclides in multiple physical forms. This requires: (i) external expert assumptions on the expected accident-specific ratios of different nuclides (otherwise the number of estimated variables would be computationally infeasible); and (ii) modification of the observation operator in order to calculate observed gamma dose rate as a sum of cloudshine and groundshine dose rates in the case of radionuclides with deposition. These modifications will increase the computational complexity; however, this is becoming a minor issue with development of computers.

\section{Acknowledgements}

Research is supported by the Ministry of the Interior of the Czech Republic (project VG20102013018).

\section{References}

[1] V. Šmídl and R. Hofman, Tracking of atmospheric release of pollution using unmanned aerial vehicles, Atmospheric Environment (2013), in press, DOI: 10.1016/j.atmosenv.2012.10.054.

[2] H. Eleveld, Y. Kok and C. Twenhofel, Data assimilation, sensitivity and uncertainty analyses in the Dutch nuclear emergency management system: a pilot study, International Journal of Emergency Management 4 (2007), pp. 551-563.

[3] H. Jeong, E. Kim, K. Suh, W. Hwang, M. Han and H. Lee, Determination of the source rate released into the environment from a nuclear power plant, Radiation protection dosimetry 113 (2005), pp. 308-313.

[4] D. Q. Zheng, J. K. C. Leung, B. Y. Lee and H. Y. Lam, Data assimilation in the atmospheric dispersion model for nuclear accident assessments, Atmospheric Environment 41 (2007), pp. 2438-2446.

[5] G. Johannesson, B. Hanley and J. Nitao, Dynamic Bayesian models via Monte Carlo-An introduction with examples, Lawrence Livermore National Laboratory, (2004).

[6] J. Y. Kim, H. K. Jang and J. K. Lee, Source reconstruction of unknown model parameters in atmospheric dispersion using dynamic Bayesian inference, Progress in Nuclear Science and Technology 1 (2011), pp. 460-463.

[7] P. Pecha and R. Hofman, Calculations of external irradiation from radioactive plume in the early stage of nuclear accident, International Journal of Environment and Pollution 1-4 (2012), pp. 420-430.

[8] A. Doucet, N. De Freitas and N. Gordon, Sequential Monte Carlo methods in practice, Springer Verlag, (2001), ISBN 0387951466.

[9] R. Hofman, Application of advanced data assimilation methods in off-site consequence assessment, dissertation, Faculty of Nuclear Sciences and Physical Engineering, CTU, Prague, (2011). 